Pacific Journal of Mathematic

Pus an D TMES 


\title{
PLUS AND TIMES
}

\author{
R. F. Arnold and A. P. Morse
}

Postulated here are very general but nevertheless concrete notions of plus and times. The generality achieved lessens the need for abstract linear spaces. The addition and multiplication are universally associative and commutative and multiplication rather widely distributes over addition.

The addable entities are of three general types: numbers, functions, and classes. The numbers are the finite complex numbers along with certain infinities. The addable functions are addable valued nonvacuous functions. The addable classes are free of numbers and ordered pairs; they are structured nonvacuous classes of addable entities. Many addable classes arise, as in $\$ 5$, as congruence classes from an equivalence relation. In particular, the usual complex Lebesgue classes are among the addable classes.

Foremost in our minds when deciding which classes would be addable and how they should be added and multiplied were certain traditional equivalence classes. Suppose for example we regard as equivalent any two complex finite valued functions which are on the unit interval and are there almost everywhere equal. We notice here: that the sum and the product of two corresponding equivalence classes can be calculated combinatorially ; nonzero scalar multiplication can be achieved in the natural combinatorial manner; whereas zero scalar multiplication of a class is the combinatorial difference of the class with itself. Accordingly the algebra of these classes does not depend on how they arose. In keeping with this we wish to stress that, in general, the addability of classes will depend solely on the behavior of their members and not on how the classes arose. To smooth our path we insist at the outset that each numerical valued function in an addable class be finite valued. If we meet a tentative candidate of finite value somewhere and of infinite value somewhere then we recommend its restriction in domain to the points where it is of finite value.

The set theory we use is given by J. L. Kelley in the appendix to his General Topology, except that his ordered pair is to be revised in the spirit of $\S 3$. The empty set is 0 , the universe is $U$, and it is important to realize that

- This particular addition permits cancellation, but combinatorial addition for nonvacuous closed subsets of the unit interval does not. 


$$
f(x)=\mathrm{U}
$$

whenever $f$ is a function and $x \notin \operatorname{domain} f$. It is also important to realize that

$$
\{x\}=\mathrm{U} \quad \text { whenever } \quad x \notin \mathrm{U} .
$$

We agree

$$
\operatorname{scsr} x=x \cup\{x\}
$$

We agree:

$$
\begin{aligned}
& 0=\text { the empty set } \\
& 1=\operatorname{scsr} 0 \\
& 2=\operatorname{scsr} 1 \\
& 3=\operatorname{scsr} 2
\end{aligned}
$$

We agree that $\omega$ is the set of all these natural numbers.

Of course 0 is a function with domain $0=0$ and

$$
0(x)=\mathrm{U} \text { for each } x \text {. }
$$

If the result, in a given instance, of an operation is $U$, then, in that instance, we think of the operation as unperformable. We shall have

$$
x+\mathrm{U}=\mathrm{U}=x \cdot \mathrm{U}
$$

To guide the reader we suggest:

the pure imaginary unit $=\mathrm{i}$;

the extended real number system $=\mathrm{rl}$;

the real finite numbers $=\mathrm{rf}$;

real infinity $=\infty$;

$-\infty=\infty^{\prime} ;$

the finite complex numbers $=\mathrm{kf}$;

complex infinity $=\$$;

the directed infinities $=$ dinfin;

the complex extension $=\mathrm{kt}$. 
In matters of order and arithmetic we assign:

completely traditional properties to $\mathrm{rf}$ and $\mathrm{kf}$; widely accepted arithmetical properties to the extended complex plane,

$$
\mathrm{kf} \cup\{\phi\}
$$

generally accepted properties of order, but not yet of arithmetic, to rl.

The foundation of our whole construction is $\mathrm{kf}$.

Traditionally addition and multiplication have involved $\infty$ and \$. We shall uphold this tradition but since

$$
\mathrm{i} \cdot(\mathrm{i} \cdot \infty)=(\mathrm{i} \cdot \mathrm{i}) \cdot \infty=-\infty,
$$

we are forced to recognize that $\mathrm{i} \cdot \infty$ must be palpable or, in other words, different from $U$.

The calculation

$$
\infty+(\infty-\infty)=(\infty+\infty)-\infty=\infty-\infty
$$

persuades us that

$$
\infty-\infty=\mathrm{U}
$$

the possibility that

$$
\infty-\infty=\infty \text { or } \quad \infty-\infty=-\infty
$$

leads us to the contradiction that

$$
\infty=-\infty .
$$

We think of the directed infinities as the rays emanating from the origin of $\mathrm{kf}$ and we think of $\phi$ as $\mathrm{kf}$. Addition of infinities is $\mathrm{U}$ unless both infinities are the same directed infinity; a directed infinity added to itself is itself; a scalar added to an infinity is the infinity; multiplication of infinities is combinatorial; nonzero scalar multiplication of an infinity is to be achieved in the obvious combinatorial manner; zero multiplication of an infinity is $\mathrm{U}$.

The addition and multiplication of nonvacuous functions is given in 1.14.3 and 1.14.4. We want to stress here that 0 is never the answer.

We close the paper in $\S 6$ with a brief discussion of linear spaces.

Although unpublished, all of the results in $\$ 2$ have been for many years known to A. P. Morse and his students.

We are grateful to Trevor J. McMinn for helpful suggestions. 
1. Postulates. We regard the expressions

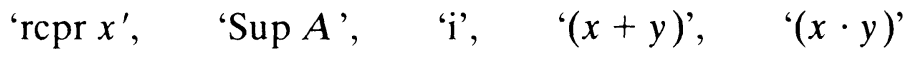

as those forms which primitively enter our postulates. In these

$$
\text { 'rcpr', 'Sup', 'i', ‘+', ‘.' }
$$

are constants. In $A M^{1}$ we have shown that these constants can be so fixed by definitions that all our postulates become theorems. Some of our postulates first emerged from a long inductive construction described in $A M$. Notable among these are: $1.6,1.12 .1,1.13 .2,1.14$. In $\$ 2$ of $A M$ we have shown that, in a reasonable sense, our postulates uniquely describe our addition and multiplication.

We think it noteworthy that Postulates $1.0-1.3$ do not involve plus and times.

The postulates of structure which involve the actual makeup of members of $\mathrm{kt}$ are: 1.2, 1.9.0, 1.9.1, 1.10.2, 1.10.3, 1.12.3.

In 1.6.3 we have addable classes in mind.

If $x$ and $y$ are complex Lebesgue classes for the unit interval then, in the sense of 1.6.5,

$$
x=+=y,
$$

and $x=y$ in the event that $x \cap y \neq 0$. Because of this we find 4.5 and the sentence preceding 4.6 of particular interest.

$$
\begin{array}{rl}
1.0 & \text { Postulated Definitions. } \\
.0 & (x \leqq y) \text { iff Sup }\{x y\}=y \neq U \\
.1 & (x<y) \text { iff } x \leqq y \text { and } x \neq y . \\
.2 & \mathrm{rl}=\{x: x \leqq x\} . \\
.3 & \infty=\text { Sup rl. } \\
.4 & \infty^{\prime}=\text { Sup } 0 \\
.5 & \mathrm{rf}=\left\{x: \infty^{\prime}<x<\infty\right\} \\
.6 & \mathrm{rfp}=\{x: 0<x<\infty\} \\
.7 & \mathrm{rp}=\{x: 0<x\} .
\end{array}
$$

\subsection{Postulates.}

$.0 A \subset \mathrm{rl}$ iff $\operatorname{Sup} A \in \mathrm{rl}$ iff $\operatorname{Sup} A \neq \mathrm{U} \neq \mathrm{rl}$.

.1 If $A \subset \mathrm{rl}$ and $t \in \mathrm{rl}$ then

$$
\operatorname{Sup} A \leqq t
$$


iff

$$
y \leqq t \quad \text { whenever } \quad y \in A
$$

.2 If $\{x y\}=A \subset$ rl then $\operatorname{Sup} A \in A$.

1.2 Postulates.

$.0 \infty=\{x: 0 \leqq x<\infty\}$.

$.1 \omega \subset \mathrm{rf}$.

1.3 Postulated Definitions.

$.0 \phi=\operatorname{rcpr} 0$.

$.1 \mathrm{kt}=\{x: \operatorname{rcpr} x \neq \mathrm{U}\}$.

.2 infin $=\{x: \operatorname{rcpr} x=0\}$.

$.3 \mathrm{kf}=\mathrm{kt} \cap \sim$ infin.

$.4 \operatorname{dinfin}=\operatorname{infin} \cap \sim\{\phi\}$.

1.4 Postulated Definition. $(x-y)=x+(\mathrm{i} \cdot \mathrm{i}) \cdot y$.

1.5 Postulated Definitions.

$.0(A++B)=\{x+y: x \in A$ and $y \in B\}$.

.1 $(A \cdots B)=\{x \cdot y: x \in A$ and $y \in B\}$.

$.2(A-B)=\{x-y: x \in A$ and $y \in B\}$.

1.6 Postulated Definitions.

$.0 \quad$ Reverted $x$ iff

$$
0+x=x=x+(x-x) \neq \mathrm{U}
$$

$.1 \quad$ reverted $=\{x$ : Reverted $x\}$.

$.2 \operatorname{Add}^{\prime} x$ iff $x$ is such a nonvacuous function that

$$
0+x(t)=x(t) \text { for each } t
$$

$.3 \quad \operatorname{Add}^{\prime \prime} x$ iff

$$
\begin{aligned}
& 0 \neq x \subset \text { reverted } \cap \sim \mathrm{kt}, \\
& x++(x--x) \subset x,
\end{aligned}
$$

$\{\lambda\} \cdot(x--x) \subset x--x \quad$ whenever $\quad 0 \neq \lambda \in \mathrm{kf}$.

.4 Add $x$ iff

$x \in \mathrm{kt}$ or $\operatorname{Add}^{\prime} x$ or $\operatorname{Add}^{\prime \prime} x$. 
$.5(x=+=y)$ iff

$\operatorname{Add}^{\prime \prime} x$ and $\operatorname{Add}^{\prime \prime} y$ and $x--x=y--y$.

$6 \quad(x=\cdot=y)$ iff

$$
x=+=y
$$

and

$w \cdots z=+=x$ whenever $\quad w=+=x$ and $z=+=x$.

1.7 Postulates.

$.0 \operatorname{rcpr} x \neq \mathrm{U}$ iff $\operatorname{rcpr} x \in \mathrm{kt}$ iff $x \in \mathrm{kt}$.

.1 If $x+y \neq \mathrm{U}$ then

$$
x+y \in \mathrm{kt} \quad \text { iff } \quad x \in \mathrm{kt} \text { and } y \in \mathrm{kt} .
$$

2 If $x \cdot y \neq \mathrm{U}$ then

$$
x \cdot y \in \mathrm{kt} \quad \text { iff } \quad x \in \mathrm{kt} \text { and } y \in \mathrm{kt} .
$$

.3 If $x \in \mathrm{rf}$ and $y \in \mathrm{rf}$ then

$$
x+y \in \mathrm{rf} \text { and } x \cdot y \in \mathrm{rf} .
$$

.4 If $x \in \mathrm{rp}$ and $y \in \mathrm{rp}$ then

$$
x+y \in \mathrm{rp} \quad \text { and } \quad x \cdot y \in \mathrm{rp} .
$$

1.8 Postulate. If $x \in \mathrm{rl}$ and $y \in \mathrm{rl}$ then

$$
x<y \quad \text { iff } \quad y-x \in \mathrm{rp} \text {. }
$$

\subsection{Postulates.}

.0 If $0 \neq x \in \mathrm{kt}$ then $0 \in x$.

$.1 \phi=\mathrm{kf}=\{x: x-x=0\}$.

$.2 \operatorname{dinfin}=\{z \cdot u: u=\infty$ and $0 \neq z \in \mathrm{kf}\}$.

1.10 Postulates.

.0 If $x \in \mathrm{kf}$ and $y \in$ infin then $x+y=y$.

.1 If $x \in$ infin and $y \in$ infin then

$$
x+y \neq \mathrm{U} \quad \text { iff } \quad x+y=y \quad \text { iff } \quad x=y \in \text { dinfin. }
$$

.2 If $0 \neq x \in \mathrm{kf}$ and $y \in$ infin then

$$
x \cdot y=\{x\} \cdot y .
$$

.3 If $x \in$ infin and $y \in$ infin then 


$$
x \cdot y=x \cdot y
$$

\subsection{Postulates.}

.0 $z \in \mathrm{kf}$ iff for some $x \in \mathrm{rf}$ and some $y \in \mathrm{rf}$,

$$
z=x+\mathrm{i} \cdot y
$$

.1 If $x \in \mathrm{rf}$ and $y \in \mathrm{rf}$ then

$$
x+\mathrm{i} \cdot y=0 \quad \text { iff } \quad x=0 \text { and } \quad y=0
$$

1.12 Postulates.

$.0 \quad$ If $x \in \mathrm{kt}$ then $0+x=x$.

$.10+x=1 \cdot x$.

.2 If $0 \neq x \in \mathrm{kf}$ then $x \cdot \operatorname{rcpr} x=1$.

.3 If $x \in \omega$ then $\operatorname{scsr} x=x+1$.

1.13 Postulates.

$.0 \quad x+y=y+x$ and $x \cdot y=y \cdot x$.

$.1 \quad x+(y+z)=(x+y)+z$ and $x \cdot(y \cdot z)=(x \cdot y) \cdot z$.

.2 If Reverted $z$ then $z \cdot(x+y)=z \cdot x+z \cdot y$.

1.14 Postulates.

$.0 \quad$ If $x+y \neq \mathrm{U}$ or $x \cdot y \neq \mathrm{U}$ then Add $x$.

.1 If $x \in \mathrm{kt}$ and $\operatorname{Add}^{\prime} y$ and $z$ is such a function that

$$
z(t)=x+y(t) \text { for each } t
$$

then:

$$
\begin{aligned}
& \text { if } \quad z \neq 0 \text { then } x+y=z \\
& \text { if } z=0 \text { then } x+y=\mathrm{U} .
\end{aligned}
$$

.2 If $x \in \mathrm{kt}$ and $\operatorname{Add}^{\prime} y$ and $z$ is such a function that

$$
z(t)=x \cdot y(t) \text { for each } t
$$

then:

$$
\begin{aligned}
& \text { if } \quad z \neq 0 \text { then } \quad x \cdot y=z \\
& \text { if } z=0 \text { then } x \cdot y=\mathrm{U} .
\end{aligned}
$$

.3 If $\operatorname{Add}^{\prime} x$ and $\operatorname{Add}^{\prime} y$ and $z$ is such a function that

$$
z(t)=x(t)+y(t) \text { for each } t
$$

then: 


$$
\begin{aligned}
& \text { if } \quad z \neq 0 \text { then } \quad x+y=z \\
& \text { if } z=0 \text { then } x+y=\mathrm{U} .
\end{aligned}
$$

.4 If $\operatorname{Add}^{\prime} x$ and $\operatorname{Add}^{\prime} y$ and $z$ is such a function that

$$
z(t)=x(t) \cdot y(t) \text { for each } t
$$

then:

$$
\begin{aligned}
& \text { if } z \neq 0 \text { then } x \cdot y=z \\
& \text { if } z=0 \text { then } x \cdot y=\mathrm{U} .
\end{aligned}
$$

.5 If $x \in$ infin and if $\operatorname{Add}^{\prime \prime} y$ then $x+y=\mathrm{U}=x \cdot y$.

.6 If $x \in \mathrm{kf}$ and if Add" $y$ then $x+y=\{x\}++y$.

.7 If $0 \neq x \in \mathrm{kf}$ and if $\mathrm{Add}^{\prime \prime} y$ then $x \cdot y=\{x\} \cdots y$.

$.8 \quad 0 \cdot x=x-x$.

.9 If $\mathrm{Add}^{\prime} x$ and $\mathrm{Add}^{\prime \prime} y$ then $x+y=\mathrm{U}=x \cdot y$.

.10 If $\operatorname{Add}^{\prime \prime} x$ and $\operatorname{Add}^{\prime \prime} y$ then:

$$
\begin{array}{lllll}
x+y \neq U & \text { iff } & x+y=x++y & \text { iff } & x=+=y \\
x \cdot y \neq U & \text { iff } & x \cdot y=x \cdot y & \text { iff } & x=\cdot=y .
\end{array}
$$

\subsection{Postulated Definition.}

grp $x=\{y \in$ reverted: $x \in$ reverted and $y-y=x-x\}$.

With some reluctance we add Postulate 1.16 which is a consequence of the others. The proof of 1.16 , we have in mind, is somewhat lengthy, and although independent of ordinal theory, is strongly dependent on the Axiom of Regularity and the ordered pair theory given by A. P. Morse in $2.55-2.63$ of $A$ Theory of Sets. An intuitively pleasant consequence of 1.16 is 6.4 . No other use is made of 1.16 .

\subsection{Definitions. \\ $.0-x=(\mathrm{i} \cdot \mathrm{i}) \cdot x$. \\ $.1 \quad x / y=x \cdot \operatorname{rcpr} y$.}

1.16 Postulate. grp $x \in \mathrm{U}$.

2. The complex extension. Many properties of the (extended) real numbers, $\mathrm{rl}$, can be proved using only 1.0, 1.1, and 1.2. Among these are Theorems 2.0 below.

2.0 THEOREMS.

.0 If $x \leqq y$ then $x \in \mathrm{rl}$ and $y \in \mathrm{rl}$. 
Proof. From 1.0.0 and 1.1.0 we see

$$
\operatorname{Sup}\{x y\}=y \neq \mathrm{U} \quad \text { and } \quad\{x y\} \subset \mathrm{rl} .
$$

.1 If $A \subset \mathrm{rl}$ then

$$
y \leqq \operatorname{Sup} A \quad \text { whenever } \quad y \in A .
$$

Proof. Using 1.1.0 and 1.0.2 we see

$$
\text { Sup } A \in \mathrm{rl}
$$

and so

$$
\operatorname{Sup} A \leqq \operatorname{Sup} A
$$

Because of this and 1.1.1

$$
y \leqq \operatorname{Sup} A \quad \text { whenever } \quad y \in A \text {. }
$$

.2 If $b \in \mathrm{rl}$ and $y \leqq b$ whenever $y \in A$ then $\operatorname{Sup} A \leqq b$.

Proof. Use .0 to conclude $A \subset \mathrm{rl}$. Now use 1.1.1.

.3 If $A \subset B \subset \mathrm{rl}$ then $\operatorname{Sup} A \leqq \operatorname{Sup} B$.

Proof. Use .1, .2, and 1.1.0.

$$
.4 \mathrm{rl}=\left\{x: \infty^{\prime} \leqq x \leqq \infty\right\} .
$$

Proof. After noting that

$$
0 \subset\{x\} \subset \mathrm{rl} \text { whenever } x \in \mathrm{rl} \text {, }
$$

use $1.0 .3,1.0 .4, .3,1.1 .2$, and .0.

$.5 \mathrm{rl}=\operatorname{rf} \cup\left\{\infty^{\prime} \infty\right\}$.

.6 If $x \in \mathrm{rl}$ and $y \in \mathrm{rl}$ then $x \leqq y$ or $y \leqq x$.

Proof. Use 1.1.2 and 1.0.0.

$.7 x \leqq y$ iff $x<y$ or $x=y \in \mathrm{rl}$.

.8 If $x \leqq y$ and $y \leqq z$ then $x \leqq z$.

Hint. Let $A=\{x y\} \quad$ and use 1.1.1. 
.9 If $x \leqq y$ and $y \leqq x$ then $x=y$.

$.100 \in \mathrm{rf}$ and $1 \in \mathrm{rf}$.

Proof. Use 1.2.1.

$.11 \operatorname{rfp} \subset \mathrm{rf} \subset \mathrm{rl}$.

$.12 \mathrm{rp}=\operatorname{rfp} \cup\{\infty\}$.

$.13 \operatorname{rfp} \subset \operatorname{rp} \subset \mathrm{rl}$.

2.1 THEOREMS.

$.0 \quad \mathrm{kt}=\mathrm{kf} \cup$ infin.

$.1 \quad \mathrm{kf}=\{x: 0 \cdot x=0\}$

Proof. Use 1.9.1 and 1.14.8.

$.2 \quad \mathrm{i} \in \mathrm{kf}$.

Proof. Because of $2.0 .10,1.11 .0, .0,1.7 .1$, and 1.7 .2 we have

$$
0+\mathrm{i} \cdot 1 \in \mathrm{kf}
$$

and

$$
\mathrm{i} \in \mathrm{kt} \text {. }
$$

From this, 1.13.0, 1.12.1, and 1.12 .0 we see

$$
\mathrm{i}=0+\mathrm{i}=0+\mathrm{i} \cdot 1 \in \mathrm{kf} .
$$

$.3 \omega \subset \mathrm{rf} \subset \mathrm{kf} \subset \mathrm{kt}$.

Proof. From 1.2.1 and .0 we have

$$
\omega \subset \mathrm{rf} \text { and } \mathrm{kf} \subset \mathrm{kt} \text {. }
$$

Furthermore, if $x \in \mathrm{rf}$ then, because of $2.0 .10,1.11 .0, .2, .1$, 1.7.1, and 1.12.0,

$$
\mathrm{kf} \ni x+\mathrm{i} \cdot 0=\mathrm{x}+0=x
$$

and so 
$\mathrm{rf} \subset \mathrm{kf}$.

\section{$.4 \mathrm{kf} \subset$ reverted.}

Proof. Use 1.9.1, 1.12.0, and 1.13.0.

.5 If $z \in \mathrm{kf}$ then $z \cdot(x+y)=z \cdot x+z \cdot y$.

.6 $\mathrm{i} \cdot \mathrm{i}=-1$ and $-x=-1 \cdot x$.

Proof. Use $1.17 .0,1.13 .1,1.13 .0, .2, .0,1.12 .1$, and 1.12 .0 to see

$$
-1=(\mathrm{i} \cdot \mathrm{i}) \cdot 1=\mathrm{i} \cdot(\mathrm{i} \cdot 1)=\mathrm{i} \cdot(1 \cdot \mathrm{i})=\mathrm{i} \cdot \mathrm{i} .
$$

Consequently,

$$
-1=\mathrm{i} \cdot \mathrm{i} \quad \text { and } \quad-x=(\mathrm{i} \cdot \mathrm{i}) \cdot x=-1 \cdot x .
$$

$.7 x-y=x+-y$.

$.8 x \in \mathrm{kf}$ iff $-x \in \mathrm{kf}$.

Proof. Because of .1 and .2 we see

$0 \cdot x=(0 \cdot \mathrm{i}) \cdot x=((0 \cdot \mathrm{i}) \cdot \mathrm{i}) \cdot x=0 \cdot(\mathrm{i} \cdot \mathrm{i} \cdot x)=0 \cdot-x$.

Thus

$$
0 \cdot x=0 \quad \text { iff } \quad 0 \cdot-x=0
$$

and so

$$
x \in \mathrm{kf} \quad \text { iff } \quad-x \in \mathrm{kf} \text {. }
$$

$.9-1 \cdot-1=1$.

Proof. Relying on .3, .8, .7, and .1 we have

$$
\begin{aligned}
1 & =1+0=1+-1 \cdot 0=1+-1 \cdot(1+-1) \\
& =1+-1 \cdot 1+-1 \cdot-1=(1+-1)+-1 \cdot-1 \\
& =0+-1 \cdot-1=-1 \cdot-1 .
\end{aligned}
$$

.10 If $x \in \mathrm{kt}$ then $--x=x$.

Proof. Use .6, .9, 1.12.1, and 1.12.0.

$.11 x \in \mathrm{kt}$ iff $-x \in \mathrm{kt}$. 
Proof. On the one hand, from .10 and .6, we have

$$
x=--x=-1 \cdot-x \text { whenever } \quad x \in \mathrm{kt}
$$

and so because of 1.7 .2

$$
-x \in \mathrm{kt} \quad \text { whenever } \quad x \in \mathrm{kt} \text {. }
$$

On the other hand, if $-x \in \mathrm{kt}$ then from .6

$$
-1 \cdot x \in \mathrm{kt}
$$

and so because of 1.7.2, $x \in \mathrm{kt}$.

$.12 x \in \mathrm{rf}$ iff $-x \in \mathrm{rf}$.

Proof. Assume $x \in \mathrm{rf}$ and using $.3, .8$, and 1.11.0, so choose $a \in \mathrm{rf}$ and $b \in \mathrm{rf}$ that

$$
-x=a+\mathrm{i} \cdot b
$$

and check that

$$
0=x+-x=x+(a+\mathrm{i} \cdot b)=(x+a)+\mathrm{i} \cdot b .
$$

But, because of 1.7.3

$$
x+a \in \mathrm{rf}
$$

and so, because of 1.11 .1

$$
b=0
$$

and

$$
-x=a+\mathrm{i} \cdot 0=a+0=a \in \mathrm{rf} .
$$

Consequently,

$$
-x \in \mathrm{rf} \text { whenever } x \in \mathrm{rf} .
$$

Because of this, .10, .3, and .11 we also have

$$
x=--x \in \mathrm{rf} \text { whenever }-x \in \mathrm{rf} \text {. }
$$

.13 If $x \in \mathrm{kt}$ and $y \in \mathrm{kt}$ then $-x \cdot-y=x \cdot y$.

Proof. Use .6 and .9. 
$.140<x$ iff $x \in \mathrm{rp}$.

$.150 \notin \mathrm{rp}$ and $1 \in \mathrm{rp}$.

Proof. Because of 2.0.6

$$
0<1 \text { or } 1<0
$$

and consequently, from .14 we have

$$
1 \in \mathrm{rp} \quad \text { or } 1<0 \text {. }
$$

But, because of 1.8 , if $1<0$ then

$$
-1=0+-1=0-1 \in \mathrm{rp}
$$

and so, using 1.7.4 and .9 we infer

$$
1=-1 \cdot-1 \in \mathrm{rp} \text {. }
$$

$.16-1 \neq 0$.

Proof. If $-1=0$ then because of .9 and .1 ,

$$
0=0 \cdot 0=-1 \cdot-1=1=\{0\} \neq 0 .
$$

\subsection{THEOREMS.}

$.0 \phi \in$ infin $\cap \sim \operatorname{dinfin}$.

Proof. Using 1.3.0, 1.7.0, and 2.1.3 we see

$$
\phi=\operatorname{rcpr} 0 \in \mathrm{kt} .
$$

From this, $1.9 .1,1.3 .1,1.3 .2$, and 1.3 .3 we obtain

$$
\phi=\mathrm{kf} \notin \mathrm{kf} \quad \text { and } \quad \phi \in \mathrm{kt} \cap \sim \mathrm{kf}=\text { infin. }
$$

Now use 1.3.4.

$.1 \quad$ infin $=\operatorname{dinfin} \cup\{\phi\}$.

$.2 \infty \in$ dinfin.

Proof. From 2.0.12 and 2.1.15 we have

$$
\infty \in \mathrm{rp} \quad \text { and } \quad 1 \in \mathrm{rp}
$$


and so, because of 1.7.4,

$$
1 \cdot \infty \in \mathrm{rp} \text {. }
$$

Thus

$$
1 \cdot \infty \in \mathrm{U} \text { and } 1 \cdot \infty \in \text { dinfin. }
$$

But then, because of .1, 2.1.0, and 1.7.2

$$
1 \cdot \infty \in \mathrm{kt} \text { and } \infty \in \mathrm{kt} .
$$

Employing 1.12.1 and 1.12.0 we conclude

$$
\infty=1 \cdot \infty \in \text { dinfin. }
$$

$.3 \mathrm{rp} \subseteq \mathrm{kt}$.

$.4 \infty^{\prime}=-\infty \in$ dinfin.

Proof. Since $\infty^{\prime}<0$ we see, helped by 1.8 and .3 , that

$$
0-\infty^{\prime}=0+-\infty^{\prime} \in \mathrm{rp} \quad \text { and } \quad-\infty^{\prime} \in \mathrm{rp} .
$$

But, because of 2.1 .12

$$
\infty^{\prime} \in \mathrm{rf} \quad \text { iff } \quad-\infty^{\prime} \in \mathrm{rf}
$$

and so

$$
-\infty^{\prime} \in \mathrm{rp} \cap \sim \mathrm{rf}=\{\infty\}
$$

and

$$
-\infty^{\prime}=\infty
$$

Now, using .3, 2.1.11, 2.1.10, 2.1.16, and 1.9.2 we conclude

$$
\infty^{\prime}=--\infty^{\prime}=-\infty=-1 \cdot \infty \in \text { dinfin. }
$$

$.5 \quad \mathrm{rl} \subset \mathrm{kt}$.

$.6 \quad \mathrm{rf}=\mathrm{kf} \cap \mathrm{rl}$.

$.7 x \in \mathrm{rl}$ iff $-x \in \mathrm{rl}$.

$.8 x<0$ iff $-x \in \mathrm{rp}$.

Proof. Use $2.0 .0,1.8,2.1 .7, .7, .5, .3$, and 1.12.0.

$.9 x \in$ infin iff $-x \in$ infin. 
Proof. Use 2.1.11 and 2.1.8.

.10 If $x \in$ dinfin then $x \neq-x$.

Proof. Using 1.9.2 so choose $z$ that

$$
0 \neq z \in \mathrm{kf} \text { and } x=z \cdot \infty .
$$

Accordingly,

$$
-x=-z \cdot \infty=z \cdot-\infty=z \cdot \infty^{\prime} .
$$

Now if $x=-x$ then

$$
\begin{aligned}
z \cdot \infty & =z \cdot \infty^{\prime}, \\
(\operatorname{rcpr} z) \cdot z \cdot \infty & =(\operatorname{rcpr} z) \cdot z \cdot \infty^{\prime}, \\
1 \cdot \infty & =1 \cdot \infty^{\prime} ; \\
\infty & =\infty^{\prime}<\infty .
\end{aligned}
$$

Thus $x \neq-x$.

.11 If $x \in$ infin then $x-x=\mathrm{U}=0 \cdot x$.

Proof. Use 1.10.1, .9, .10, and 1.14.8.

$.120 \cdot x=0$ iff $x \in \mathrm{kf}$ iff $0 \cdot x \in \mathrm{kt}$.

Proof. Use 1.7.2, 1.3.3, 2.1.1, and .11.

$.13 x+y \in \mathrm{kf}$ iff $x \in \mathrm{kf}$ and $y \in \mathrm{kf}$ iff $x \cdot y \in \mathrm{kf}$.

Proof. Recall first that

$$
\begin{aligned}
& 0 \cdot(x+y)=0 \cdot x+0 \cdot y, \\
& 0 \cdot(x \cdot y)=(0 \cdot x) \cdot y=x \cdot(0 \cdot y),
\end{aligned}
$$

and then use 1.7.1, 1.7.2, and .12.

.14 If $x \in$ dinfin then $x+x=x$.

$.15 \infty+\infty=\infty$.

.16 If $x \in$ infin then $x+\phi=\mathrm{U}$.

.17 If $0 \neq x \in \mathrm{kf}$ then $x \cdot \phi=\phi$. 
Proof. Using 1.10.2, 1.9.1, and .13 we see

$$
x \cdot \phi=\{x\} \cdot \operatorname{kf} \subset \mathrm{kf} .
$$

Furthermore, in the light of 1.12 .2 , if $y \in \mathrm{kf}$ then

$$
y=1 \cdot y=x \cdot \operatorname{rcpr} x \cdot y \in\{x\} \cdots \mathrm{kf}=x \cdot \phi
$$

and so

$$
\mathrm{kf} \subset x \cdot \phi .
$$

Thus $x \cdot \phi=\mathrm{kf}=\phi$.

$.18 \infty \cdot \phi=\phi$.

Proof. From 1.2.0 we learn

$$
1 \in \infty \subset \mathrm{kf} .
$$

Because of this, 1.10.3 and .17 we have

$$
\infty \cdot \phi=\infty \cdots \phi \subset \mathrm{kf}
$$

and

$$
\mathrm{kf}=\{1\} \cdots \mathrm{kf} \subset \infty \cdots \infty
$$

and so

$$
\infty \cdot \phi=\mathrm{kf}=\phi .
$$

$.19 \phi \cdot \phi=\phi$.

.20 If $0 \neq x \in \mathrm{kt}$ then $x \cdot \phi=\phi$.

.21 If $0<x$ then $x \cdot \infty=\infty$.

Proof. Using 2.1.14, 2.0.12, and 1.7.4 we see that

$$
x \cdot \infty \in \operatorname{rp}=\operatorname{rfp} \cup\{\infty\} .
$$

But, from 2.0.11, 2.2.6, and .13 we learn

$$
\text { if } x \cdot \infty \in \mathrm{rfp} \text { then } \infty \in \mathrm{kf}
$$

contrary to $2.2 .2,1.3 .3$, and 1.3.4. Thus

$$
x \cdot \infty=\infty .
$$


If $x<0$ then $x \cdot \infty=-\infty$.

Proof. Use .8, 2.1.14, and .21 to conclude

$$
-x \cdot \infty=\infty .
$$

Now, helped by $2.1 .6,2.1 .13,1.12 .0$, and 1.12 .1 , we see

$-\infty=-1 \cdot(-x \cdot \infty)=(-1 \cdot-x) \cdot \infty=(1 \cdot x) \cdot \infty=x \cdot \infty$.

.23 If $x \in \mathrm{rl}$ and $y \in \mathrm{rl}$ and $x+y \neq \mathrm{U}$ then $x+y \in \mathrm{rl}$.

.24 If $x \in \mathrm{rl}$ and $y \in \mathrm{rl}$ and $x \cdot y \neq \mathrm{U}$ then $x \cdot y \in \mathrm{rl}$.

$.250 \neq \lambda \in \mathrm{kf}$ iff $0 \neq \mathrm{rcpr} \lambda \in \mathrm{kf}$.

Proof. Use: $1.12 .2,2.2 .13,2.1 .1 ; 1.3 .1,1.3 .2,1.3 .3,1.3 .0,2.2 .0$.

3. Integrity. Some of the ordered pairs of Norbert Wiener are functions. ${ }^{2}$ Free from this blemish is the ordered pair given by A. P. Morse in 2.57.1 of $A$ Theory of Sets. It turns out that

if $x$ is an ordered pair then $0 \notin x \quad$ and $\quad\{\{0\}\} \in x$.

Using this and 1.9.0 we prove the Theorems of Integrity below. These theorems help us verify that two intuitively different things are actually different.

3.0 THEOREMS OF INTEGRITY.

.0 If $0 \neq x \in \mathrm{kt}$ then $x$ is not a relation.

Proof. Because of 1.9.0, $0 \in x$. But 0 is not an ordered pair since $\{\{0\}\} \notin 0$.

.1 If $x$ is a relation then $x$ is not an ordered pair.

Proof. If $x$ is an ordered pair then: $\{\{0\}\} \in x$; but since $\{\{0\}\} \notin\{\{0\}\}$ it follows that $\{\{0\}\}$ is not an ordered pair; thus $x$ is not a relation.

.2 If $x$ is an ordered pair then $x \notin \mathrm{kt}$.

${ }^{2}$ An example is $\{\{\{0\}\}\}$. 
Proof. Clearly 0 is not an ordered pair. Furthermore, because of 1.9.0, if $0 \neq x \in \mathrm{kt}$ then $0 \in x$ and so $x$ is not an ordered pair.

\section{Generalities.}

4.0 THEOREMS.

$.0 \quad$ If $\operatorname{Add} x$ then $x \neq \mathrm{U}$.

$.1 x+\mathrm{U}=\mathrm{U}=x \cdot \mathrm{U}$.

.2 If $x \in \mathrm{U}$ and $y \in \mathrm{U}$ then:

$$
\begin{aligned}
& \text { if } x+y \neq \mathrm{U} \text { then } x+y \in \mathrm{U} \text {; } \\
& \text { if } x \cdot y \neq \mathrm{U} \text { then } x \cdot y \in \mathrm{U} .
\end{aligned}
$$

Proof. Use 1.7.1, 1.7.2, 1.14, and set-theoretic considerations.

.3 If Add $x$ then $0+x=x=1 \cdot x$.

Proof. Use 1.6.4, 1.12.1, 1.12.0, 1.6.2, 1.14.1, 1.6.3, and 1.14.6.

$.40+x+y=x+y=1 \cdot(x+y)$ and $0+x \cdot y=x \cdot y=1 \cdot x \cdot y$.

Proof. Because of $.1, x+y=\mathrm{U}$ and $x \cdot y=\mathrm{U}$ then

$$
0+x+y=0+x \cdot y=0+\mathrm{U}=\mathrm{U}=x+y=x \cdot y .
$$

Employing 1.14.0 and .3 we infer

if $x+y \neq \mathrm{U}$ or $x \cdot y \neq \mathrm{U}$ then $\operatorname{Add} x$ and so

$$
0+x+y=(0+x)+y=x+y
$$

and

$$
1 \cdot x \cdot y=(1 \cdot x) \cdot y=x \cdot y \text {. }
$$

The desired conclusion now follows from 1.12.1.

.5 If Add $x$ then $--x=x$.

Proof. Helped by .3 we infer 


$$
--x=-1 \cdot-1 \cdot x=1 \cdot x=x .
$$

.6 If $x+y \neq \mathrm{U}$ then $\operatorname{Add}(x+y)$.

Proof. Using .4 and 1.14.0 we see

$$
(x+y)+0=0+(x+y)=x+y \neq \mathrm{U} \quad \text { and } \operatorname{Add}(x+y) .
$$

$.7 \quad$ If $x \cdot y \neq \mathrm{U}$ then $\operatorname{Add}(x \cdot y)$.

.8 If Reverted $x$ then Add $x$.

Proof. Use 1.6.0 and 1.14.0.

For those dismayed by .9 below we first agree that

$$
(x, y)=\{(1, x)(2, y)\} \text {. }
$$

Next we notice that

$\operatorname{Add}^{\prime}(x, y)$ whenever $x \in \mathrm{kt}$ and $y \in \mathrm{kt}$.

Accordingly,

$$
(3,2)+(5,7)=(8,9)
$$

.9 If $x$ is an ordered pair then $\sim \operatorname{Add} x$.

Proof. From 3.0.2 we see $x \notin \mathrm{kt}$.

From 3.0.1 we see $\sim \operatorname{Add}^{\prime} x$.

Now suppose Add"x. Since

$$
x \subset \text { reverted } \cap \sim \mathrm{kt},
$$

and, since $x$ is an ordered pair, helped by .8 we see

$$
\{\{0\}\} \in x, \quad\{\{0\}\} \in \text { reverted } \cap \sim \mathrm{kt}, \quad \operatorname{Add}\{\{0\}\} .
$$

Now $0 \in\{0\}$ and so $\{0\}$ is not an ordered pair. Consequently,

$$
\{\{0\}\} \text { is not a relation, } \sim \operatorname{Add}^{\prime}\{\{0\}\} \text {. }
$$

Furthermore

$$
\{\{0\}\}=\{1\} \not \subset \text { reverted } \cap \sim \mathrm{kt}, \quad \sim \operatorname{Add}^{\prime \prime}\{\{0\}\} .
$$

Contradictorily we conclude $\sim \operatorname{Add}\{\{0\}\}$. 
Thus $\sim \operatorname{Add}^{\prime \prime} x$ and $\sim \operatorname{Add} x$.

.10 If $x \in \mathrm{kt}$ then $\sim \operatorname{Add}^{\prime} x$ and $\sim \operatorname{Add}^{\prime \prime} x$.

Proof. Use 3.0.0, 1.6.2, 1.6.3, and 1.9.0.

.11 If $\operatorname{Add}^{\prime} x$ then $\sim \operatorname{Add}^{\prime \prime} x$.

Proof. Use 1.6.3, 1.6.2, .8, and .9.

An alternative but less enlightening proof of .11 shuns .9 but depends on 1.14 .9 and on .12 below.

.12 If $\operatorname{Add}^{\prime} x$ and $z$ and $w$ are such functions that, for $t \in \mathrm{U}$,

$$
z(t)=0 \quad \text { and } \quad w(t)=1,
$$

then

$\operatorname{Add}^{\prime} z$ and $\operatorname{Add}^{\prime} w$ and $z+x=x=w \cdot x$.

.13 If $x+y \neq \mathrm{U}$ and $\operatorname{Add}^{\prime} x$ then $\operatorname{Add}^{\prime}(x+y)$.

Proof. Use 1.7.1 and .10 to conclude $x+y \notin \mathrm{kt}$.

Choose $z$ in accordance with .12 and use .12 and 1.14 .9 to see

$$
z+(x+y)=(z+x)+y=x+y \neq \mathrm{U}
$$

and so

$$
\sim \operatorname{Add}^{\prime \prime}(x+y)
$$

Consequently, because of 1.14.0, $\operatorname{Add}^{\prime}(x+y)$.

.14 If $x \cdot y \neq \mathrm{U}$ and $\operatorname{Add}^{\prime} x$ then $\operatorname{Add}^{\prime}(x \cdot y)$.

.15 If $x+y \neq \mathrm{U}$ and $\operatorname{Add}^{\prime \prime} x$ then $\operatorname{Add}^{\prime \prime}(x+y)$.

Proof. Use 1.7.1 and .10 to conclude $x+y \notin \mathrm{kt}$.

Again choose $z$ in accordance with .12 and use .12, 1.14.9, and .1 to see

$$
z+(x+y)=(z+x)+y=\mathrm{U}+y=\mathrm{U}
$$

and hence, because of $.12, \sim \operatorname{Add}^{\prime}(x+y)$. 
Because of 1.14.0 the desired conclusion is now at hand.

.16 If $x \cdot y \neq \mathrm{U}$ and $\operatorname{Add}^{\prime \prime} x$ then $\operatorname{Add}^{\prime \prime}(x \cdot y)$.

.17 If Add" $y$ then $x--y=x++-y$.

.18 If Add $x$ and $0 \neq \lambda \in \mathrm{kf}$ then $\lambda \cdot x \neq \mathrm{U}$.

Proof. Helped by 1.12 .2 and .1 we see

$$
\mathrm{U} \neq x=1 \cdot x=(\lambda \cdot \operatorname{rcpr} \lambda) \cdot x=(\operatorname{rcpr} \lambda) \cdot(\lambda \cdot x)
$$

and $\lambda \cdot x \neq \mathrm{U}$.

.19 If $0 \neq \lambda \in \mathrm{kf}$ and $\operatorname{Add}^{\prime} x$ then $\operatorname{Add}^{\prime}(\lambda \cdot x)$.

Proof. Use .14 and .18.

.20 If $\operatorname{Add}^{\prime \prime} x$ then $x=+=x$.

.21 If $x=+=y$ then $y=+=x$.

.22 If $x=+=y$ and $y=+=z$ then $x=+=z$.

.23 If $x=\cdot=y$ then $y=\cdot=x$.

.24 If $x=\cdot=y$ and $y=\cdot=z$ then $x=\cdot=z$.

A theorem is not obtained from .20 by replacing ' + ' by ' $\because$ '. Nevertheless if $x=\cdot=y$ then $x=\cdot=x$.

.25 If Add"x then $x=+=-x$.

Proof. Use .18 and .16 to conclude Add" $-x$. Then use .17. .26 If $x=+=y$ then $\mathrm{U} \neq x-y=x--y$.

Proof. Use .25, 1.14.10, and .17.

.27 If $\lambda \in \operatorname{kf}$ then $\lambda \cdot(x-x)=\lambda \cdot x-\lambda \cdot x=x-x$.

Proof. Using 2.1.5 and 1.14 .8 we see 


$$
\begin{aligned}
\lambda \cdot(x-x) & =\lambda \cdot x-\lambda \cdot x=0 \cdot(\lambda \cdot x)=(0 \cdot \lambda) \cdot x \\
& =0 \cdot x=x-x .
\end{aligned}
$$

.28 If $\lambda \in \mathrm{kf}$ and $\operatorname{Add}^{\prime \prime} x$ then $\operatorname{Add}^{\prime \prime}(\lambda \cdot x)$.

Proof. Use .26 and .16 to conclude

$$
0 \cdot x=x-x \neq \mathrm{U} \text { and } \operatorname{Add}^{\prime \prime}(0 \cdot x)
$$

and hence because of .18 and .16

$$
\operatorname{Add}^{\prime \prime}(\lambda \cdot x)
$$

.29 If $\operatorname{Add}^{\prime \prime} x$ and $\lambda \in \mathrm{kf}$ then $x=+=\lambda \cdot x$.

Proof. Helped by .28, .26, and .27 we see

$$
\lambda \cdot x-\lambda \cdot x=\lambda \cdot x-\lambda \cdot x=x-x=x--x
$$

and

$$
x=+=\lambda \cdot x
$$

.30 If $x \subset$ reverted and $x++x-x \subset x$ then

$$
x++x-x=x .
$$

Proof. Evidently if $u \in x$ then

$$
u=u+u-u \in x++x--x
$$

and hence $x \subset x++x--x$.

.31 If $\operatorname{Add}^{\prime \prime} x$ then $x+x-x=x$.

Proof. Helped by $1.14 .8, .29,1.14 .10, .26$, and .30 we see

$$
\begin{aligned}
x+x-x & =x+0 \cdot x=x++0 \cdot x \\
& =x++(x-x)=x++x--x=x .
\end{aligned}
$$

.32 If $\operatorname{Add}^{\prime \prime} x$ then Reverted $x$.

.33 If $x+y \neq \mathrm{U}$ and $\operatorname{Add}^{\prime \prime} x$ then $(x+y)=+=x$.

.34 If $x \cdot y \neq \mathrm{U}$ and $\operatorname{Add}^{\prime \prime} x$ and $\operatorname{Add}^{\prime \prime} y$ then $(x \cdot y)=\cdot=x$. 
.35 If $x \cdot y \neq \mathrm{U}$ and $\operatorname{Add}^{\prime \prime} x$ then $(x \cdot y)=+=x$.

\subsection{TheOREMS.}

.0 If Reverted $x$ and Reverted $y$ and $x+y \neq \mathrm{U}$ then $\operatorname{Reverted}(x+y)$.

.1 If Reverted $x$ and Reverted $y$ and $x \cdot y \neq \mathrm{U}$ then $\operatorname{Reverted}(x \cdot y)$.

.2 If Reverted $x$ and $\lambda \in \operatorname{kf}$ then $\operatorname{Reverted}(\lambda \cdot x)$.

.3 If $x \in$ reverted $\cap \sim \mathrm{kt}$ then Add"grp $x$.

.4 If Reverted $x$ and $x-x=y-y$ then $x+y \neq \mathrm{U}$.

Proof. Helped by 1.6.0 and 4.0.1 we see

$$
\mathrm{U} \neq x=x+(x-x)=x+(y-y)=(x+y)-y
$$

and

$$
x+y \neq \mathrm{U} \text {. }
$$

4.2 Theorem. If $\operatorname{Add}^{\prime \prime} x$ and $\operatorname{Add}^{\prime \prime} y$ and $0 \neq x++y$ then

$$
\operatorname{Add}^{\prime \prime}(x++y) \text {. }
$$

4.3 Theorem of CAncellation. If $x+y=\eta$ and Reverted $x$ then $(x+\eta)-(x+\eta)=\eta-\eta$.

Proof. Using 1.13 and 1.14 .8 we have

$$
\begin{aligned}
(x+\eta)-(x+\eta) & =0 \cdot(x+\eta) \\
& =0 \cdot x+0 \cdot \eta \\
& =0 \cdot x+0 \cdot(x+y) \\
& =0 \cdot x+0 \cdot x+0 \cdot y \\
& =(0+0) \cdot x+0 \cdot y \\
& =0 \cdot x+0 \cdot y \\
& =0 \cdot(x+y) \\
& =0 \cdot \eta \\
& =\eta-\eta .
\end{aligned}
$$


4.4 Theorem of Shift. If $\operatorname{Add}^{\prime \prime} x$ and $\eta \in x-x$ then there is such $a \xi \in x$ that $\xi-\xi=\eta-\eta$.

Proof. So choose $u, v$, and $\xi$ that

$$
u \in x, \quad v \in x, \quad \eta=u-v
$$

and

$$
\xi=u+\eta
$$

Then, from 1.6.3 and 4.3 we have

$$
u \in \text { reverted and } \xi-\xi=\eta-\eta \text {. }
$$

Now, helped by 4.0 .2 and 4.0 .30 we see

$$
\begin{aligned}
(u+u-v)-u= & (u+u-u)-v \\
= & u-v=\eta \in \mathrm{U}, \\
u+u-v \neq \mathrm{U}, \quad & u+u-v \in \mathrm{U},
\end{aligned}
$$

and

$$
\xi=u+\eta=u+u-v \in x++x--x=x .
$$

4.5 TheOREm. If $x=+=y$ and $x \subset y$ then $x=y$.

Proof. Assume $z \in y$. Then since $y \subset$ reverted,

$$
z=z+z-z
$$

Thus,

$$
z-z \neq \mathrm{U} \text { and } z-z \in y--y=x--x \text {. }
$$

Consequently, we so choose $u \in x$ and $v \in x$ that

$$
z-z=u-v
$$

and infer

$$
z=z+z-z=z+u-v=z-v+u .
$$

But, since $x \subset y$,

$v \in y \quad$ and $\quad z-v \neq \mathrm{U}$ and $z-v \in y--y=x--x$.

We now so choose $r \in x$ and $s \in x$ that

$$
z-v=r-s
$$


and conclude

$$
z=z-v+u=r-s+u=r+u-s \in x++x--x \subset x .
$$

Thus $z \in x$. The arbitrary nature of $z$ assures us that $x=y$.

In connection with 4.5 we note that if

$$
\xi=\{\{(0,0)\}\{(1,0)\}\}, \quad \eta=\{\{(0,0)\}\{(1,1)\},
$$

then

$$
\xi=+=\eta, \quad \xi \cap \eta=\{\{(0,0)\}\} \neq 0, \quad \xi \neq \eta .
$$

4.6 THEOREMS.

.0 If $x \in \mathrm{kf}$ then $\operatorname{grp} x=\mathrm{kf}$.

.1 If $\operatorname{Add}^{\prime} x$ then $\operatorname{grp} x=\{y: y$ is on domain $x$ and for each $t \in \operatorname{domain} x, y(t) \in \operatorname{grp} x(t)\}$.

Here grp $x$ is a Cartesian product.

.2 If $\operatorname{Add}^{\prime \prime} x$ then $\operatorname{grp} x=\{y: y=+=x\}$.

4.7 Conjecture. If $\operatorname{Add}^{\prime \prime} x, s \in x$, and $t \in \operatorname{grp} s$, then there is a $y$ for which

$$
t \in y=+=x
$$

\section{Equivalence relations.}

5.0 Definition. Relation" $R$ iff $R$ is such an equivalence relation that:

$$
\text { domain } R \subset \text { reverted } \cap \sim \mathrm{kt} \text {; }
$$

$(s+u, t+v) \in R$ whenever $s, t, u$, and $v$ are such that

$$
(s, t) \in R, \quad(u, v) \in R, \quad s+u \neq \mathrm{U} \neq t+v
$$

$(\lambda \cdot s, \lambda \cdot t) \in R$ whenever $s, t$, and $\lambda$ are such that

$$
(s, t) \in R, \quad \lambda \in \mathrm{kf} .
$$

5.1 Theorem. If Relation" $R$ and $0 \neq x=\{u:(u, a) \in R\}$ then $\operatorname{Add}^{\prime \prime} x$. 
We give the proof in 4 parts.

Part $0 . \quad 0 \neq x \subset$ reverted $\cap \sim$ kt.

Part 1. $x++x--x \subset x$.

Proof. Suppose $u \in x, v \in x, w \in x$, and

$$
u+v-w \in x++x--x .
$$

Thus $(u, a) \in R,(v, a) \in R,(w, a) \in R,(-w,-a) \in R$, and

$$
(u+v-w, a)=(u+v-w, a+a-a) \in R .
$$

Consequently,

$$
u+v-w \in x
$$

Part 2. $\{\lambda\} \cdots(x--x) \subset x--x$ whenever $0 \neq \lambda \in \mathrm{kf}$.

Proof. Suppose $u \in x, v \in x, 0 \neq \lambda \in \mathrm{kf}$, and

$$
\lambda \cdot(u-v) \in\{\lambda\} \cdots(x--x) .
$$

Thus

$.0 \quad(u, a) \in R, \quad(\lambda \cdot u, \lambda \cdot a) \in R, \quad$ and $\quad(-\lambda \cdot v,-\lambda \cdot a) \in R$ and because of 4.0.27

$$
\begin{aligned}
\mathrm{U} \neq \lambda \cdot(u-v) & =\lambda \cdot u-\lambda \cdot v \\
& =\lambda \cdot(u+u-u)-\lambda \cdot v \\
& =\lambda \cdot u+\lambda \cdot(u-u)-\lambda \cdot v \\
& =\lambda \cdot u+u-u-\lambda \cdot v \\
& =(u+\lambda \cdot u-\lambda \cdot v)-u .
\end{aligned}
$$

Thus

$$
u+\lambda \cdot u-\lambda \cdot v \neq \mathrm{U}
$$

and hence because of .0

$$
\begin{aligned}
(u+\lambda \cdot u-\lambda \cdot v, a) & =(u+\lambda \cdot u-\lambda \cdot v, a+a-a) \\
& =(u+\lambda \cdot u-\lambda \cdot v, a+\lambda \cdot a-\lambda \cdot a) \in R .
\end{aligned}
$$

Accordingly, 


$$
u+\lambda \cdot u-\lambda \cdot v \in x
$$

and, because of .1

$$
\lambda \cdot(u-v) \in x--x .
$$

Part 3. Add" $x$.

Proof. Use 1.6.3 and Parts 0, 1, and 2.

5.2 Theorem. If Relation" $R, \quad 0 \neq x=\{u:(u, a) \in R\}$, and $0 \neq \lambda \in \mathrm{kf}$ then

$$
\lambda \cdot x=\{u:(u, \lambda \cdot \alpha) \in R\}
$$

Proof. Use $2.2 .25,1.12 .2$, and 5.0 to check that

$$
(u, a) \in R \quad \text { iff } \quad(\lambda \cdot u, \lambda \cdot a) \in R
$$

Now use 5.1 and 1.14.7.

5.3 THEOREM. If Relation" $R$,

$$
\begin{aligned}
& 0 \neq x=\{u:(u, a) \in R\}, \\
& 0 \neq y=\{u:(u, b) \in R\},
\end{aligned}
$$

and

corresponding to each $s \in x$ there is such $a w \in y$ that

$$
w-w=s-s,
$$

then:

$$
\begin{aligned}
& .0 \quad(a-a, b-b) \in R ; \\
& .1 \quad x--x \subset y--y .
\end{aligned}
$$

Proof. Recall first that if $q \in x \cup y$ then $q+q-q=q$ and $q-q \in U$.

So choose $w \in y$ that

$$
w-w=a-a .
$$

Thus

$$
\begin{aligned}
&(w, b) \in R, \quad(-w,-b) \in R, \quad \text { and } \\
&(a-a, b-b)=(w-w, b-b) \in R .
\end{aligned}
$$


The proof of .0 is now complete. Next assume

$$
s \in x, \quad t \in x, \quad s-t \in x--x
$$

and note that

$$
(s, a) \in R, \quad(t, a) \in R, \quad(s-t, a-a) \in R,
$$

and because of .0 ,

$$
(s-t, b-b) \in R .
$$

Now so choose $w$ that

$$
(w, b) \in R \quad \text { and } \quad w-w=s-s .
$$

Hence

.4

$$
\begin{aligned}
\mathrm{U} \neq s-t & =s+s-s-t \\
& =s+w-w-t \\
& =(w+s-t)-w,
\end{aligned}
$$

and so

$$
w+s-t \neq \mathrm{U} .
$$

Because of this, .2, and .3

$$
(w+s-t, b)=(w+s-t, b+b-b) \in R .
$$

Accordingly,

$$
w+s-t \in y
$$

and, because of .4

$$
s-t \in y--y .
$$

The proof of .1 is now complete.

5.4 THEOREM. If Relation" $R$,

$$
\begin{aligned}
& 0 \neq x=\{u:(u, a) \in R\}, \\
& 0 \neq y=\{u:(u, b) \in R\},
\end{aligned}
$$

and

corresponding to each sand $\operatorname{tin} x \cup y$ is such a w that 


$$
(w, t) \in R \quad \text { and } \quad w-w=s-s,
$$

then

$$
x=+=y \text {. }
$$

5.5 TheOREM. If Relation" $R, a+b \neq \mathrm{U}$,

$$
\begin{aligned}
0 \neq x & =\{u:(u, a) \in R\}, \\
0 \neq y & =\{u:(u, b) \in R\}, \\
z & =\{u:(u, a+b) \in R\},
\end{aligned}
$$

and

corresponding to each sand $t$ in $x \cup y \cup z$ is such a w that

$$
(w, t) \in R \quad \text { and } \quad w-w=s-s
$$

then

$$
x+y=z
$$

Proof. Helped by 5.4, 4.0.33, and 1.14 .10 we see

$$
\begin{gathered}
x=+=y, \quad x=+=z, \\
x+y=x++y, \quad x=+=(x+y), \\
z=+=(x+y) .
\end{gathered}
$$

Evidently

$$
x+y=x++y \subset z
$$

and consequently, because of .0 and 4.5 ,

$$
x+y=z \text {. }
$$

For many applications a more pleasant equivalence relation is available.

5.6 Definition. Relation $+R$ iff $R$ is such an equivalence relation that for each $s, t, u, v$ in domain $R$ and each $\lambda \in \mathrm{kf}$ :

$$
\text { domain } R \subset \text { reverted } \cap \sim \mathrm{kt} \text {; }
$$

if $(s, t) \in R$ and $(u, v) \in R$ then

$$
(s+u, t+v) \in R \quad \text { and } \quad(\lambda \cdot s, \lambda \cdot t) \in R
$$

for some $w$ 


$$
\begin{gathered}
(w, t) \in R \quad \text { and } w-w=s-s ; \\
\text { grp } s \subset \text { domain } R .
\end{gathered}
$$

5.7 Theorem. If Relation $+R$ then Relation" $R$.

5.8 Theorem. If Relation" $R, D=\operatorname{domain} R$, and

$$
\operatorname{grp} x=D \text { whenever } x \in D \text {, }
$$

then

$$
\text { Relation }+R
$$

Proof. Use 5.0, 5.6, and 4.1.4.

5.9 ThEOREM. If Relation $+R$,

$$
\begin{aligned}
& 0 \neq x=\{u:(u, a) \in R\}, \\
& 0 \neq y=\{u:(u, b) \in R\},
\end{aligned}
$$

then

$$
x=+=y
$$

Proof. Use 5.4 and 5.6.

5.10 TheOREM. If Relation $+R$,

$$
\begin{aligned}
0 \neq x & =\{u:(u, a) \in R\}, \\
0 \neq y & =\{u:(u, b) \in R\}, \\
z & =\{u:(u, a+b) \in R\},
\end{aligned}
$$

then

$$
x+y=z
$$

Proof. Use 5.5, 5.6, and 5.7.

5.11 Lemma. If Relation $+R, s \in$ domain $R, t \in \operatorname{domain} R$, and $(s-t, t-t) \in R$ then

$$
(s, t) \in R
$$

Proof. Evidently, because of 5.6,

$$
(s-t+t, t-t+t) \in R, \quad(s+t-t, t) \in R
$$

Also, by 5.6 and 5.3, 


$$
\begin{aligned}
& (s-s, t-t) \in R, \quad(s+s-s, s+t-t) \in R, \\
& (s, s+t-t) \in R .
\end{aligned}
$$

Consequently, by transitivity,

$$
(s, t) \in R .
$$

5.12 THEOREM. If Relation $+R$ and $b \in y=+=x=$ $\{u:(u, a) \in R\}$ then

$$
y=\{u:(u, b) \in R\} .
$$

Proof. Assume

$$
z=\{u:(u, b) \in R\}
$$

and divide the rest of the proof into 3 parts.

Part 0. $y \subset$ domain $R$.

Proof. If $t \in y$ then, helped by 5.2, 5.10, and 5.6, we see

$$
\begin{gathered}
t-t \in y-y=x-x=\{u:(u, a-a) \in R\}, \\
(t-t, a-a) \in R, \\
t-t \in \text { domain } R, \\
t \in \operatorname{grp}(t-t) \subset \text { domain } R, \\
t \in \text { domain } R .
\end{gathered}
$$

Part 1. $y \subset z$.

Proof. Assume $t \in y$. Using Part 0, 5.3.0, and 5.11 we see

$$
\begin{gathered}
t-b \in y-y=x-x=\{u:(u, a-a) \in R\}, \\
(t-b, a-a) \in R, \\
(t-b, b-b) \in R, \\
(t, b) \in R, \\
t \in z .
\end{gathered}
$$

Part 2. $y=z$.

Proof. Use 5.9 and 4.5. 
5.13 Conjecture. If Relation" $R, \quad b \in y=+=x=$ $\{u:(u, a) \in R\}, z=\{u:(u, b) \in R\}$, and

corresponding to each $s$ and $t$ in $x \cup y \cup z$ is such a $w$ that

$$
(w, t) \in R \quad \text { and } \quad w-w=s-s,
$$

then

$$
y=z
$$

5.14 Definition. Relation $R$ iff Relation $+R$ and for each $s, t, u$, and $v$ in domain $R$ :

if $\quad(s, t) \in R \quad$ and $\quad(u, v) \in R \quad$ then $\quad(s \cdot u, t \cdot v) \in R$

if $(u, s \cdot t) \in R$ then, for some $p$ and $q$

$$
u=p \cdot q, \quad(p, s) \in R, \quad \text { and } \quad(q, t) \in R .
$$

5.15 Lemma. If Relation $\cdot R$,

$$
\begin{aligned}
0 \neq x & =\{u:(u, a) \in R\}, \\
0 \neq y & =\{u:(u, b) \in R\}, \\
z & =\{u:(u, a \cdot b) \in R\},
\end{aligned}
$$

then

$$
x \cdot y=z \text {. }
$$

5.16 THEOREM. If Relation $\cdot R$,

$$
\begin{aligned}
0 \neq x & =\{u:(u, a) \in R\}, \\
0 \neq y & =\{u:(u, b) \in R\}, \\
z & =\{u:(u, a \cdot b) \in R\},
\end{aligned}
$$

then

$$
x \cdot y=z
$$

Proof. According to 5.14, 5.9, and 5.15

$$
x=+=y \quad \text { and } \quad x \cdot y=z .
$$

Now suppose

$$
w=+=x \text { and } v=+=x
$$


and note that $0 \neq w$ and $0 \neq v$. Assume $c \in w$ and $d \in v$ and use 5.12 to see that

$$
w=\{u:(u, c) \in R\} \quad \text { and } \quad v=\{u:(u, d) \in R\}
$$

and so, because of 5.15

$$
w \cdot v=\{u:(u, c \cdot d) \in R\} .
$$

Because of this and 5.9

$$
w \cdot v=+=x
$$

and consequently from 1.6.6 we have

$$
x=\cdot=y \text {. }
$$

Now use 1.14.10.

5.17 THEOREM. If $J$ is the unit interval,

$$
D=\{x: x \text { is on } J \text { to } \mathrm{kf}\},
$$

$R$ is the set of points of the form $(x, y)$ where $x \in D$ and $y \in D$ and

$$
x(t)=y(t) \text { for almost all } t \in J,
$$

then

$$
\text { Relation } \cdot R \text {. }
$$

Of course 5.10 and 5.16 apply. Here the equivalence classes are Lebesgue classes of the coset type.

5.18 THEOREM. If $J$ is the unit interval, and $\Delta$ consists of those functions $x$ for which

$$
\text { domain } x \subset J \text { and } \text { range } x \subset \mathrm{kf} \text {, }
$$

$R$ is the set of points of the form $(x, y)$ where $x \in \Delta$ and $y \in \Delta$ and

$$
x(t)-y(t)=0 \quad \text { for almost all } \quad t \in J,
$$

then

Relation $\cdot R$ 
Again 5.10 and 5.16 apply but now the equivalence classes are Lebesgue classes which are not of the coset type.

5.19 THEOREM. If $P$ consists of those functions $x$ for which

$$
0 \in \text { domain } x \in U \text { and range } x \subset \mathrm{kf} \text {, }
$$

$R$ is the set of points of the form $(x, y)$ where $x \in P$ and $y \in P$ and

$$
x(0)=y(0)
$$

then

$$
\text { Relation } \cdot R \text {. }
$$

Still again 5.10 and 5.16 apply but now each equivalence class has the power of the universe.

\section{Linear spaces.}

6.0 Definition. $S$ is a linear space iff

$$
S \subset \text { reverted }
$$

and, for each $x \in S$, each $y \in S$, and each $\lambda \in \mathrm{kf}$,

$$
0 \cdot x=0 \cdot y, \quad x+y \in S, \text { and } \lambda \cdot x \in S .
$$

6.1 Theorem. $S$ is a linear space iff for each $x \in S$, each $y \in S$, each $\lambda \in \mathrm{kf}$, and each $\mu \in \mathrm{kf}$,

$$
\begin{aligned}
& 0 \cdot x=0 \cdot y, \\
& x+y \in S, \\
& \lambda \cdot x \in S, \\
& (\lambda+\mu) \cdot x=\lambda \cdot x+\mu \cdot x .
\end{aligned}
$$

In connection with 6.1 recall $1.13,2.1 .4,1.14 .0$ and 4.0.3. In mild departure from custom we have insured that 0 is a linear space.

6.2 Theorem. grp $x$ is a linear space.

6.3 Theorem. If $S$ is a linear space and $x \in S$ then $S \subset \operatorname{grp} x$.

6.4 Theorem. If $S$ is a linear space then $S \in \mathrm{U}$. 
It interests us that if $\xi \in \operatorname{grp} x, \eta \in \operatorname{grp} x, \xi \cdot \eta \in \operatorname{grp} x$, then grp $x$ is a commutative ring.

6.5 REMARKs. Our linear spaces have a certain integrity. By a lengthy set-theoretic argument it can be shown, for example, that if $S$ is a linear space and $x \in S$ then no point in range $x$ belongs to $S$. Because of this and $\S 3$ it is possible, with proper choice of $x$ and $y$, for

to consistently be either an instance of multiplication of scalars, or function evaluation, or composition, or a rather general inner product, or matrix multiplication, or the left application of a matrix to a vector, or the right application of a matrix to a vector. The operation we here have in mind is not universally associative.

We shall now be a bit more specific. If $x$ and $y$ are matrices and $y$ does not properly receive $x$ then

$$
(x y)=0
$$

If $x$ is a function and $y \in$ domain $x$ then (xy) is the value of $x$ at $y$. For convenience we agree that $x$ is spanic iff $x$ is a function, domain $x$ is included in some linear space, and domain $x$ is not a subset of

$$
\{j \in \omega: 1 \leqq j\} .
$$

If $x$ is spanic and $y$ is a function for which $y \notin$ domain $x$, and $C$ is the composition of $x$ and $y$, then:

$$
\begin{array}{ll}
\text { if } \quad C \neq 0 \text { then } \quad(x y)=C \\
\text { if } C=0 \text { then } \quad(x y)=\{(0,0)\} .
\end{array}
$$

If $n \in \omega$ and $x$ and $y$ are on

$$
\{j \in \omega: 1 \leqq j \leqq n\}
$$

then

$$
(x y)=\sum_{j=1}^{n}\left(x_{j} \cdot y_{j}\right)
$$

It can easily happen here that

$$
(x y) \in \sim \mathrm{kt} .
$$


Because of the integrity of linear spaces it is easy to see that if $x$ is spanic and $y$ is a function for which

$$
\text { range } y \cap \text { domain } x \neq 0 \text {, }
$$

then (xy) is the composition of $x$ and $y$. Since the domain of a matrix is a relation, it follows from 4.0.9 that no matrix is spanic. We do not object to the convention that

$$
(x y=(x y))
$$

In this connection we note that if $x=0$ and $y=1$, then

$$
\{x y\} \neq\{(x y)\}=\{0\} .
$$

We wish also to point out that if $a$ is a nonvacuous matrix then

$$
0 a=0 \neq 0 \cdot a \text {. }
$$

Received April 10, 1975.

California State University-Fresno

AND

The UNIVERSITY OF CALIFORNIA-BERKELEY 


\section{PACIFIC JOURNAL OF MATHEMATICS}

\section{EDITORS}

RICHARD ARENS (Managing Editor)

University of California

Los Angeles, California 90024

\author{
R. A. Beaumont \\ University of Washington \\ Seattle, Washington 98105
}

\section{J. DugunduI}

Department of Mathematics University of Southern California Los Angeles, California 90007

D. Gilbarg and J. Milgram

Stanford University

Stanford, California 94305

\section{ASSOCIATE EDITORS}

E. F. BECKENBACH
B. H. NeumanN

F. Wolp
K. YoshidA

\section{SUPPORTING INSTITUTIONS}

UNIVERSITY OF BRITISH COLUMBIA

CALIFORNIA INSTITUTE OF TECHNOLOGY

UNIVERSITY OF CALIFORNIA

MONTANA STATE UNIVERSITY

UNIVERSITY OF NEVADA

NEW MEXICO STATE UNIVERSITY

OREGON STATE UNIVERSITY

UNIVERSITY OF OREGON

OSAKA UNIVERSITY

\author{
UNIVERSITY OF SOUTHERN CALIFORNIA \\ STANFORD UNIVERSITY \\ UNIVERSITY OF HAWAII \\ UNIVERSITY OF TOKYO \\ UNIVERSITY OF UTAH \\ WASHINGTON STATE UNIVERSITY \\ UNIVERSITY OF WASHINGTON \\ AMERICAN MATHEMATICAL SOCIETY
}

The Supporting Institutions listed above contribute to the cost of publication of this Journal, but they are not owners or publishers and have no responsibility for its contents or policies.

Mathematical papers intended for publication in the Pacific Journal of Mathematics should be in typed form or offset-reproduced (not dittoed). double spaced with large margins. Underline Greek letters in red, German in green, and script in blue. The first paragraph or two must be capable of being used separately as a synopsis of the entire paper. Items of the biblography should not be cited there unless absolutely necessary, in which case they must be identified by author and Journal, rather than by item number. Manuscripts, in duplicate, may be sent to any one of the four editors. Please classify according to the scheme of Math. Reviews, Index to Vol. 39. All other communications should be addressed to the managing editor, or Elaine Barth, University of California, Los Angeles, California, 90024.

100 reprints are provided free for each article, only if page charges have been substantially paid. Additional copies may be obtained at cost in multiples of 50 .

The Pacific Journal of Mathematics is issued monthly as of January 1966. Regular subscription rate: $\$ 72.00$ a year (6 Vols., 12 issues). Special rate: $\$ 36.00$ a year to individual members of supporting institutions.

Subscriptions, orders for back numbers, and changes of address should be sent to Pacific Journal of Mathematics, 103 Highland Boulevard, Berkeley, California, 94708.

PUBLISHED BY PACIFIC JOURNAL OF MATHEMATICS, A NON-PROFIT CORPORATION Printed at Jerusalem Academic Press, POB 2390, Jerusalem, Israel.

Copyright (C) 1976 Pacific Journal of Mathematics All Rights Reserved 


\section{Pacific Journal of Mathematics}

\section{Vol. 64, No. $2 \quad$ June, 1976}

Richard Fairbanks Arnold and A. P. Morse, Plus and times............. 297

Edwin Ogilvie Buchman and F. A. Valentine, External visibility ......... 333

R. A. Czerwinski, Bonded quadratic division algebras.............. 341

William Richard Emerson, Averaging strongly subadditive set functions in unimodular amenable groups. II .........................

Lynn Harry Erbe, Existence of oscillatory solutions and asymptotic behavior for a class of third order linear differential equations ............. 369

Kenneth R. Goodearl, Power-cancellation of groups and modules........ 387

J. C. Hankins and Roy Martin Rakestraw, The extremal structure of locally compact convex sets ...................................

Burrell Washington Helton, The solution of a Stieltjes-Volterra integral

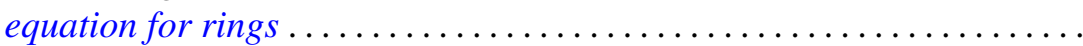

Frank Kwang-Ming Hwang and Shen Lin, Construction of 2-balanced

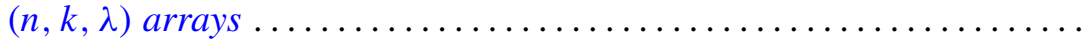

Wei-Eihn Kuan, Some results on normality of a graded ring ... 455

Dieter Landers and Lothar Rogge, Relations between convergence of series and convergence of sequences ......................... 465

Lawrence Louis Larmore and Robert David Rigdon, Enumerating immersions and embeddings of projective spaces ................

Douglas C. McMahon, On the role of an abelian phase group in relativized problems in topological dynamics..................

Robert Wilmer Miller, Finitely generated projective modules and TTF classes...

Yashaswini Deval Mittal, A class of isotropic covariance functions ...

Anthony G. Mucci, Another martingale convergence theorem ...

Joan Kathryn Plastiras, Quasitriangular operator algebras ...

John Robert Quine, Jr., The geometry of $p\left(S^{1}\right) \ldots \ldots \ldots$. 